\title{
Immunotherapy may protect cancer patients from SARS-CoV-2 infection: a single-center retrospective analysis
}

\author{
Maria Antonietta Isgròn ${ }^{2}$, Maria Grazia Vitale ${ }^{2}$, Egidio Celentano ${ }^{3}$, Flavia Nocerino ${ }^{3}$, Giuseppe Porciello ${ }^{3}$, \\ Marcello Curvietto², Domenico Mallardo², Concetta Montagnese², Luigi Russo ${ }^{2}$, Nicoletta Zanaletti ${ }^{4}$, \\ Antonio Avallone ${ }^{4}$, Matilde Pensabene ${ }^{5}$, Michelino De Laurentiis ${ }^{5}$, Sara Centonze ${ }^{6}$, Sandro Pignata ${ }^{6}$, \\ Lucia Cannella ${ }^{7}$, Alessandro Morabito ${ }^{8}$, Francesco Caponigro ${ }^{9}$, Gerardo Botti ${ }^{10}$, Giuseppe Valentino Masucci ${ }^{11}$, \\ Diana Giannarelli ${ }^{12}$, Ernesta Cavalcanti ${ }^{1}$ and Paolo Antonio Ascierto ${ }^{2^{*}}$ (1)
}

\begin{abstract}
Coronavirus disease 2019 (COVID-19) global pandemic has created unique challenges to healthcare systems throughout the world. Ensuring subjects' safety is mandatory especially in oncology, in consideration of cancer patients' particular frailty. We examined the proportion of severe acute respiratory syndrome coronavirus 2 (SARS-CoV-2) IgM and/ or IgG positive subjects in three different groups from Istituto Nazionale Tumori - IRCCS "Fondazione G. Pascale" in Naples (Campania region, Italy): cancer patients treated with Innovative Immunotherapy (Immune Checkpoint Inhibitors, ICls), cancer patients undergoing standard Chemotherapies (CHTs) and healthcare providers. 9 out of 287 (3.1\%) $\mathrm{ICls}$ patients resulted positive, with a significant lower percentage in respect to $\mathrm{CHTs}$ patients (39 positive subjects out of $598,6.5 \%)(p=0.04)$. There was no statistically significant difference between ICls cohort and healthcare providers, 48 out of 1050 resulting positive (4.6\%). Performing a Propensity Score Matching based on gender and tumor stage, the effect of treatment on seropositivity was analyzed through a regression logistic model and the ICls treatment resulted to be the only protective factor significantly $(p=0.03)$ associated with positivity (odds ratio-OR: $0.41 ; 95 \%$ confidence interval- $\mathrm{Cl}$ 0.18-0.91). According to these preliminary data, ICls would appear to be a protective factor against the onset of COVID-19 infection.
\end{abstract}

Keywords: Immunotherapy, Chemotherapy, SARS-CoV-2 infections, ICls, SARS-CoV-2 seropositivity

\section{Introduction}

During coronavirus disease 2019 (COVID-19) global pandemic, the oncologist community is debating about two main issues: the first is to guarantee continuity of care for cancer patients, the second is about patients' safety. A recent paper published in Nature Medicine has stated that determining the incidence of COVID-19

\footnotetext{
${ }^{*}$ Correspondence: p.ascierto@istitutotumori.na.it; paolo.ascierto@gmail.com ${ }^{2}$ Unit of Melanoma, Cancer Immunotherapy and Innovative Therapy, Istituto Nazionale Tumori - IRCCS - Fondazione G. Pascale, Naples, Italy Full list of author information is available at the end of the article
}

through the use of large-scale serological testing is a priority, caring for patients with cancer [1].

A pooled meta-analysis of 11 retrospective studies reported that nearly $2.0 \%$ of patients with COVID-19 were affected by cancer, showing an increased susceptibility to COVID-19 infection [2]. Nevertheless, data from a French trial carried out by Barlesi et al. from Gustave Roussy Cancer Center involving 137 cancer patients diagnosed with severe acute respiratory syndrome coronavirus 2 (SARS-CoV-2) showed a rate of infection similar to that of global population [3].

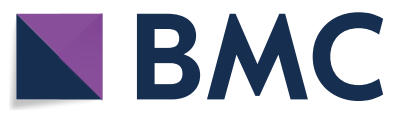

(c) The Author(s) 2021. This article is licensed under a Creative Commons Attribution 4.0 International License, which permits use, sharing, adaptation, distribution and reproduction in any medium or format, as long as you give appropriate credit to the original author(s) and the source, provide a link to the Creative Commons licence, and indicate if changes were made. The images or other third party material in this article are included in the article's Creative Commons licence, unless indicated otherwise in a credit line to the material. If material is not included in the article's Creative Commons licence and your intended use is not permitted by statutory regulation or exceeds the permitted use, you will need to obtain permission directly from the copyright holder. To view a copy of this licence, visit http://creativeco mmons.org/licenses/by/4.0/. The Creative Commons Public Domain Dedication waiver (http://creativecommons.org/publicdomain/ zero/1.0/) applies to the data made available in this article, unless otherwise stated in a credit line to the data. 
Data regarding COVID-19 infection severity in cancer patients are discordant and limited to date. First reports recorded more severe symptoms and worse outcomes in these patients $[4,5]$. Zhang et al. reported that cancer seems to be associated most frequently with severe SARS-CoV-2 infection in a subset of lung cancer patients [6]. On the contrary, Barlesi et al. didn't find any evidence that COVID-19 is more lethal or aggressive in cancer patients [3].

Another concern is about the relationship between type of oncological therapy and the development of COVID19 infection. While standard Chemotherapies (CHTs) are generally associated with immunosuppression and increased risk of infection, the role of Innovative Immunotherapy (Immune Checkpoint Inhibitors, ICIs) in promoting severe SARS-CoV-2 infection is in fact source of debate. As we know, Innovative Immunotherapy, based on ICIs, activates the immune system against cancer, but the inflammatory storm of the activated immune system could be directed against other organs (interstitial pneumonia occurs in $2.5-5 \%$ of ICIs in monotherapy and in $7-10 \%$ of combination therapy). The risk of overlapping syndromes due to SARS-CoV-2 infection with similar pathogenesis is theoretic, but it cannot be excluded. Few data support this idea. A retrospective study of Memorial Sloan Kettering Cancer Center enrolled 423 patients with COVID-19 infection and concomitant cancer. Among these patients $31(7 \%)$ were treated with ICIs. The study demonstrated that ICIs treatment within 90 days is a predictor for hospitalization and severe disease: OR (odds ratio) of 2.53 (95\% confidence interval-CI 1.18-5.67), $\mathrm{p}=0.017$ in univariate analysis; OR of 2.84 (95\% CI 1.246.72), $p=0.013$ in multivariate analysis [7]. Otherwise first results of an international, registry-based, cohort study of Thoracic cancERs internAtional coVid 19 cOLlaboraTion (TERAVOLT) were reported by Garassino [8]. The study enrolled 200 patients with thoracic cancer and COVID-19 infection diagnosis since 26 March until 12 April 2020 in 21 countries around the world. To note, 147 (73.5\%) patients were on treatment, 34 of which (23.1\%) on ICIs and 20 (13.6\%) on CHTs/ICIs combination. Preliminary analysis reported that no cancer treatment, including ICIs, was associated to an increased risk of death or hospitalization. Moreover, since it would seem that PD-1/PDL-1 pathway is an escape mechanism for some pathogens in preclinical models and the use of anti-PDL-1 could increase the clearance of some viruses, like influenza virus [9], it could be hypothesized that ICIs may play a protective role against SARS-CoV-2 infection.

In order to keep everybody safe, many cancer centers have reorganized the management of patients, avoiding gatherings and prioritizing treatments as well as according to scientific societies [1].
In our experience at Istituto Nazionale Tumori IRCCS "Fondazione G. Pascale" in Naples, a tightened program of health surveillance for patients and healthcare providers has been planned and performed, in order to early detect and promptly quarantine subjects with SARS-CoV-2 infection. Reorganization of internal management has allowed to ensure an adequate protection for cancer patients afferent to our Institute. COVID-19 screening performed with rapid serological tests revealed that ICIs could protect cancer patients from SARS$\mathrm{CoV}-2$ infection.

\section{Materials and methods}

From 30 March 2020 to 15 May 2020, 885 cancer patients admitted to Istituto Nazionale Tumori - IRCCS "Fondazione G. Pascale" in Naples (Italy) and 1050 healthcare providers working in the same hospital were tested for specific SARS-CoV-2 Immunoglobulins IgG and IgM, in accordance with a tightened program of internal health surveillance, addressed to ensure an adequate protection for frail cancer patients. In all cases entering the clinic during the period, the analyses were performed. Patients underwent a triage survey in which the proper questions to the symptoms were asked.

Patients' cohorts included 287 subjects suffering from melanoma undergoing ICIs (anti-PD-1 or anti-CTLA-4) and 598 patients undergoing CHTs.

Patients with hematological malignancies were excluded, in order to avoid eventual false negative results, due to their state of immunodepression/immunosuppression. Their particular condition, in fact, associated with low titers of immunoglobulins, makes difficult for qualitative immunochromatographic tests to identify such low titers of specific immunoglobulins, albeit present.

Whole blood samples from patients and healthcare providers arrived to the Laboratory of Istituto Nazionale Tumori - IRCCS "Fondazione G. Pascale" Cancer Center and were centrifuged immediately. Plasma samples obtained after centrifugation were tested for specific SARS-CoV-2 Immunoglobulins IgG and IgM, using Leccurate - SARS-CoV-2 Antibody Test kit (Lepu Medical Technology - Beijing - Co., Ltd.), within $1 \mathrm{~h}$, upon recommendations. The assay is a qualitative colloidal gold immunochromatography based on the principle of antigen-antibody reaction and immunoassay technique: it is a qualitative method for which data regarding cutoffs are not available to define positivity, nor related units of measurement, nor the detection limit. The producer declares that for the detection of sensitivity reference material, the positive detection rate should be no less than 90\%; for the detection of negative reference material, the negative detection rate should be $100 \%$; for the detection of positive reference material the positive 
detection rate should be $100 \%$. The positivity to IgG and/ or IgM is defined only visually, if the relative colored band appears near the areas where the relative antihuman mouse antibodies are pre-adhered, after binding any antibodies to a recombinant protein of the COVID19 marked with colloidal gold particles. All tests were performed by the same laboratory technician and the results interpreted by the same operator, in order to avoid inter-operator and inter-observer variability. Both healthcare workers were blinded to group allocation of patients (ICIs and CHTs) and personnel. Patients and healthcare professionals were considered positive for SARS-CoV-2 infection if either IgG or IgM, or both, resulted positive at least at one determination. Data on age, cancer stage, Eastern Cooperative Oncology Group (ECOG) Performance score, line of treatment, symptoms, lymphopenia, leukopenia were collected for most of patients.

\section{Statistical analysis}

Statistical analysis was performed by using the Statistical Package for Social Science (SPSS Inc., Chicago, IL, USA), version 26.0. Categorical variables were displayed as frequencies and the appropriate non-parametric tests $\left(x^{2}\right.$ test) were used to assess significance of the differences between groups; $p$ values $<0.05$ were considered statistically significant. In order to assess association between cancer treatment (ICIs and CHTs), SARS-CoV-2 IgG and/or IgM seropositivity and clinical characteristics of patients' sample-gender, dichotomized variables of age (less than 70 years versus equal or more than 70 years), cancer stage (IV versus I, II, III), ECOG performance status ( 0 versus $1,2,3,4)$, lymphopenia and leukopenia conditions (yes or not), $\chi^{2}$ tests were performed and OR with $95 \%$ CI were computed.

A Propensity Score Matching was implemented in order to form two comparable subsets. Random choice of matched patients based on Nearest Neighbor Matching was implemented.

Multiple linear logistic regression was used to adjust treatment effect by other factors. A forward stepwise selection based on Wald statistics was adopted with 0.05 and 0.10 enter and remove significance level respectively.

\section{Results}

885 cases with cancer diagnosis admitted to Istituto Nazionale Tumori - IRCCS "Fondazione G. Pascale" in Naples (Italy) from 30 March 2020 to 15 May 2020, undergoing different oncological treatments and 1050 healthcare providers were tested for SARS-CoV-2 serology (demographic data are reported in Table 1 ).

287 patients with a diagnosis of melanoma undergoing ICIs treatment ( 154 male/133 female) had a median age of 62 (IR-interquartile range: 53-73) years (Table 1 ). 598 CHTs patients came from breast $(\mathrm{N}=183)$, thoracopulmonary $(\mathrm{N}=16)$, uro-gynaecological $(\mathrm{N}=118)$, head and neck $(\mathrm{N}=16)$, sarcoma $(\mathrm{N}=30)$, gastro-intestinal $(\mathrm{N}=235)$ units, respectively, with an overall median age of 63 (IR: 54-71) years and an overall distribution of 207 male/391 female subjects (Table 1). 1050 healthcare providers (491 male/559 female) had a median age of 47 (IR: 38-55) years (Table 1 ).

Patients undergoing ICIs and CHTs carried out serological test for immunoglobulins. 9 (3.1\%, 95\% CI $1.1-$ $5.1)$ of the patients treated with ICIs and $39(6.5 \%, 95 \% \mathrm{CI}$ 4.5-8.5) of the patients treated with CHTs had a positive

Table 1 Patients' and Healthcare providers' demographic data

\begin{tabular}{|c|c|c|c|c|c|c|}
\hline \multirow{2}{*}{$\begin{array}{l}\text { Oncology unit } \\
\text { category }\end{array}$} & \multirow{2}{*}{ Treatment } & \multirow{2}{*}{$\begin{array}{l}\text { Number of } \\
\text { patients }\end{array}$} & \multicolumn{2}{|c|}{ Male/female patients } & \multicolumn{2}{|c|}{ Median age (interquartile range) (years) } \\
\hline & & & Number & Ratio & Subgroups & Groups \\
\hline Melanoma & ICIs & 287 & $154 / 133$ & 1.2 & - & $62(53-73)$ \\
\hline Breast & CHTs & 183 & $0 / 183$ & 0.0 & $57(49-67)$ & \\
\hline Thoraco-pulmonary & CHTs & 16 & $12 / 4$ & 3.0 & $69(58-70)$ & \\
\hline Uro-gynaecological & CHTs & 118 & $35 / 83$ & 0.4 & $65(55-72)$ & \\
\hline Head-neck & CHTs & 16 & $9 / 7$ & 1.3 & $61(57-66)$ & 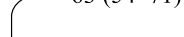 \\
\hline Sarcoma & CHTs & 30 & $14 / 16$ & 0.9 & $54(46-63)$ & \\
\hline Gastro-intestinal & CHTs & 235 & $137 / 98$ & 1.4 & $67(59-74)$ & \\
\hline $\begin{array}{l}\text { Total CHTs "non } \\
\text { melanoma" patients }\end{array}$ & CHTs & 598 & $207 / 391$ & 0.5 & - & $63(54-71)$ \\
\hline \multirow{2}{*}{ Category } & & \multirow{2}{*}{$\begin{array}{l}\text { Number of } \\
\text { cases }\end{array}$} & \multicolumn{2}{|c|}{ Male/female subjects } & \multirow{2}{*}{\multicolumn{2}{|c|}{ Median age (interquartile range) (years) }} \\
\hline & & & Number & Ratio & & \\
\hline Healthcare providers & & 1050 & $491 / 559$ & 0.9 & - & $47(38-55)$ \\
\hline
\end{tabular}


serological test for immunoglobulins, $\mathrm{p}=0.04$ (Pearson's $\chi^{2}$ test) (Table 2). At the same time, 1050 healthcare providers underwent serological test for immunoglobulins, 48 of which resulted positive $(4.6 \%, 95 \%$ CI 3.3-5.9) (Table 2). The cohort of employees was compared with the cohort of patients undergoing ICIs and no significant difference was found (Pearson's $X^{2}$ test) (Table 2). According to these data, we observed the higher incidence of immunoglobulins in patients treated with CHTs when compared to patients treated with ICIs or health care workers (albeit with some overlap in the confidence intervals).

In the analysis of association all the other clinical variables were significantly associated to cancer treatment except age (Table 3), while SARS-CoV-2 IgG and/or IgM positivity was associated with gender $(\mathrm{p}<0.001)$ and cancer stage $(\mathrm{p}=0.03)$ (Table 4$)$. Data about symptoms recorded in the triage survey did not present relevant statistical differences between the cohort of patients.

To better investigate the association between cancer treatment and seropositivity, we performed a Propensity Score Matching based on gender and tumor stage. We obtained two groups of equal size $(n=287$, each) in which sex and tumor stage were perfectly balanced. The effect of treatment on seropositivity in this matched subset was analyzed through a regression logistic model and the ICIs treatment resulted to be the only protective factor significantly $(\mathrm{p}=0.03)$ associated with positivity (OR: 0.41 ; $95 \%$ CI $0.18-0.91)$ together with female gender
Table 3 Association between type of treatment and clinical variables

\begin{tabular}{|c|c|c|c|c|}
\hline Clinical variables & $\mathrm{ICls}$ & CHTs & $\mathrm{n}$ & $p$-value* \\
\hline \multicolumn{5}{|l|}{ SARS-CoV2 lgG/lgM } \\
\hline $\lg G$ and/or $\lg M+$ & 9 & 39 & 885 & 0.04 \\
\hline $\lg G$ and $\lg M-$ & 278 & 559 & & \\
\hline \multicolumn{5}{|l|}{ Gender } \\
\hline Male & 154 & 207 & 885 & $<0.001$ \\
\hline Female & 133 & 391 & & \\
\hline \multicolumn{5}{|l|}{ Age (years) } \\
\hline$<70$ & 194 & 416 & 885 & n.s \\
\hline$\geq 70$ & 93 & 182 & & \\
\hline \multicolumn{5}{|l|}{ Cancer stage } \\
\hline$|-|||$ & 38 & 97 & 804 & 0.05 \\
\hline IV & 249 & 420 & & \\
\hline \multicolumn{5}{|c|}{ ECOG performance status } \\
\hline 0 & 244 & 265 & 793 & $<0.001$ \\
\hline $1-4$ & 41 & 243 & & \\
\hline \multicolumn{5}{|l|}{ Lymphopenia } \\
\hline Yes & 2 & 90 & 755 & $<0.001$ \\
\hline No & 256 & 407 & & \\
\hline \multicolumn{5}{|l|}{ Leukopenia } \\
\hline Yes & 0 & 91 & 764 & $<0.001$ \\
\hline No & 258 & 415 & & \\
\hline
\end{tabular}

ICIs Immune Checkpoint Inhibitors Treatment, CHTs chemotherapy treatments, $n$ number of patients, $n . s$ not statistically significant

* The $p$-values represent $x^{2}$ tests of independence indicating associations between type of treatment and categorical clinical variables (statistically significant $\mathrm{p}<0.05$ )

Table 2 SARS-CoV-2 IgM and/or lgG positivity percentages of patients (divided into oncology units) and healthcare providers

\begin{tabular}{|c|c|c|c|c|c|c|c|c|c|c|}
\hline \multirow{2}{*}{\multicolumn{2}{|c|}{ Oncology unit category }} & \multirow[t]{2}{*}{ Treatment } & \multirow{2}{*}{\multicolumn{2}{|c|}{$\begin{array}{l}\text { Number of } \\
\text { patients }\end{array}$}} & \multicolumn{2}{|c|}{ IgM and IgG negative } & \multicolumn{3}{|c|}{ IgM and/or lgG positive } & \multirow[t]{2}{*}{ p-value } \\
\hline & & & & & $\begin{array}{l}\text { Number of } \\
\text { patients }\end{array}$ & $\begin{array}{l}\text { Percentage of } \\
\text { patients }\end{array}$ & $\begin{array}{l}\text { Numb } \\
\text { patier }\end{array}$ & $\begin{array}{l}\text { er of } \\
\text { ts }\end{array}$ & $\begin{array}{l}\text { Percentage of } \\
\text { patients }\end{array}$ & \\
\hline \multicolumn{2}{|l|}{ Melanoma } & $\mathrm{ICls}$ & 287 & & 278 & 96.9 & 9 & & 3.1 & - \\
\hline \multicolumn{2}{|l|}{ Breast } & $\mathrm{CHTS}$ & 183 & & 165 & 90.2 & 18 & & 9.8 & - \\
\hline \multicolumn{2}{|l|}{ Thoraco-pulmonary } & $\mathrm{CHTS}$ & 16 & & 15 & 93.8 & 1 & & 6.3 & \\
\hline \multicolumn{2}{|l|}{ Uro-gynaecological } & $\mathrm{CHTS}$ & 118 & & 114 & 96.6 & 4 & & 3.4 & \\
\hline \multicolumn{2}{|l|}{ Head-neck } & $\mathrm{CHTS}$ & 16 & & 16 & 100.0 & 0 & & 0.0 & \\
\hline \multicolumn{2}{|l|}{ Sarcoma } & $\mathrm{CHTS}$ & 30 & & 27 & 90.0 & 3 & & 10.0 & \\
\hline \multicolumn{2}{|l|}{ Gastro-intestinal } & $\mathrm{CHTS}$ & 235 & & 222 & 94.47 & 13 & & 5.5 & \\
\hline \multicolumn{2}{|c|}{ Total CHTs "non melanoma" patients } & $\mathrm{CHTS}$ & 598 & & 559 & 93.5 & 39 & & 6.5 & $0.04^{*}$ \\
\hline \multirow[t]{2}{*}{ Category } & Number of cases & \multicolumn{4}{|c|}{ IgM and IgG negative } & \multicolumn{4}{|c|}{ IgM and/or IgG positive } & $p$-value \\
\hline & & \multicolumn{2}{|c|}{ Number of subjects } & \multicolumn{2}{|c|}{ Percentage of subjects } & Number of su & bjects & \multicolumn{2}{|c|}{ Percentage of subjects } & \\
\hline Healthcare providers & 1050 & 1002 & & 95.4 & & 48 & & 4.6 & & $0.29^{* *}$ \\
\hline
\end{tabular}

ICls Immune Checkpoint Inhibitors Treatment, CHTs chemotherapy treatments

* Comparison ICls vs. CHTs patients' SARS-CoV-2 IgM and/or lgG positivity percentages (statistically significant) (Pearson's $X^{2}$ test was used to identify differences in the proportions of individuals between two categories)

** Comparison ICls patients' vs. Healthcare Providers' SARS-CoV-2 IgM and/or lgG positivity percentages (not statistically significant) (Pearson's $X^{2}$ test was used to identify differences in the proportions of individuals between two categories) 
Table 4 Association between lgG and/or IgM seropositivity and clinical variables

\begin{tabular}{|c|c|c|c|c|c|c|}
\hline Main features & $\lg$ and/or lgM+ & $\lg G$ and $\lg M-$ & $\mathrm{n}$ & $p$-value* & OR & $95 \% \mathrm{Cl}$ \\
\hline \multicolumn{7}{|l|}{ Gender } \\
\hline Male & 11 & 350 & 885 & 0.01 & 0.41 & $0.21-0.82$ \\
\hline Female & 37 & 487 & & & & \\
\hline \multicolumn{7}{|l|}{ Age (years) } \\
\hline$<70$ & 31 & 579 & 885 & n.s & 1.23 & $0.67-2.26$ \\
\hline$\geq 70$ & 17 & 258 & & & & \\
\hline \multicolumn{7}{|l|}{ Cancer stage } \\
\hline $0-|| \mid$ & 12 & 123 & 804 & 0.04 & 0.48 & $0.24-0.97$ \\
\hline IV & 30 & 639 & & & & \\
\hline \multicolumn{7}{|c|}{ ECOG performance status } \\
\hline 0 & 25 & 484 & 793 & n.s & 1.16 & $0.61-2.20$ \\
\hline $1-4$ & 16 & 268 & & & & \\
\hline \multicolumn{7}{|l|}{ Lymphopenia } \\
\hline Yes & 5 & 87 & 755 & n.s & 1.06 & $0.41-2.79$ \\
\hline No & 34 & 629 & & & & \\
\hline \multicolumn{7}{|l|}{ Leukopenia } \\
\hline Yes & 6 & 85 & 764 & n.s & 1.38 & $0.56-3.36$ \\
\hline No & 33 & 640 & & & & \\
\hline
\end{tabular}

$n$ number of patients, OR odds ratio, n.s not statistically significant

*The $p$-values represent $x^{2}$ tests of independence indicating associations between SARS-CoV-2 IgG and/or IgM seropositivity and categorical clinical variables (statistically significant $\mathrm{p}<0.05$ )

being a significant $(\mathrm{p}=0.01)$ unfavorable item (OR: 2.87; 95\% CI 1.29-6.41).

\section{Discussion}

The concept of frailty has become increasingly recognized as one of the most important issues in health care and health outcomes and is of particular importance in patients with cancer. Frailty is a complex state of diminished physiologic reserve that results in increased vulnerability to stressors, leading to adverse health outcomes [10]. One important clinical question is how to manage patients who need anticancer therapy, including ICIs during these conditions.

As reported by Poortmans, the more recent larger studies have evidenced that none of the anti-cancer therapeutic regimens may affect neither the rate of severe COVID-19 nor the mortality rate in cancer patients [11]. On the contrary, the study conducted by Robilotti at the Memorial Sloan Kettering Cancer Center, has highlighted, specifically for ICIs, an association with increased intensive care unit admission rate, but not death rate [7]. So, Vivarelli et al. conclude that the question remains still debated: is ICIs administration harmful or beneficial for cancer patients during the COVID-19 pandemic [12]? Their hypothesis is that using ICIs in cancer patients during the pandemic does not harm and might be a game-changer. Based on the positive effect that ICIs have towards T-cell reactivation against cancer cells, as well as virus-infected cells, they conclude that ICIs administration may not represent a risk for cancer patients during this pandemic and can be suggested as protective for cancer patients who are infected by the SARS-CoV-2 [12]. According to this hypothesis, our preliminary data suggest a possible protective effect of ICIs against SARS-CoV-2 infection onset. In our patients' cohorts, individuals undergoing treatment with ICIs presented significant lower proportion of IgG and/or IgM positivity in respect to patients treated with $\mathrm{CHTs}$, ICIs seeming to be a protective factor against COVID-19 infection. At the same time, ICIs cohort presented a risk of SARS-CoV-2 infection similar to that of healthcare providers, showing that ICIs treatment is able to restore an adequate immunocompetent status in cancer patients. When a Propensity Score Matching based on gender and tumor stage was performed and effect of treatment on seropositivity analyzed, the ICIs treatment was demonstrated to be a significantly protective factor.

Overall, according to our findings, we may carry forward our hypothesis according which Innovative Immunotherapy based on ICIs treatment could protect cancer patients from COVID-19 infection. Nevertheless, further studies and epidemiologic data are needed to better define risk population among cancer patients and fill clinical and preclinical gaps to provide strong evidence-based 
therapeutic guidance and physiopathological insights on the possible immune intersection between COVID-19 disease and cancer therapy.

A registered clinical study currently ongoing (NCT04343144) conducted on severe patients affected by COVID-19 requiring hospitalization in conventional unit or in intensive care unit will give us additional insights about the possible role of ICIs in protection from SARSCoV-2 infection, uncovering the differential efficacy to eradicate infection in COVID-19 patients treated either with anti-PD-1 antibody nivolumab in association with standard care protocol, or with standard care alone.

\section{Abbreviations}

COVID-19: Coronavirus disease 2019; SARS-CoV-2: Severe acute respiratory syndrome coronavirus 2; ICls: Immune Checkpoint Inhibitors; CHTs: Standard Chemotherapies; OR: Odds ratio; 95\% Cl: 95\% Confidence intervals.

\section{Acknowledgements}

None.

Provenance and peer review

Not commissioned; externally peer reviewed.

\section{Authors' contributions}

PAA and ECa conceived, designed and supervised the project. MAI, MGV, MC, NZ, AV, MP, MDL, SC, SP, LC, AM, FC and GB assisted with data collection. MAI, DM and LR performed the tests. MAI, FN, GP, CM, ECe and DG performed the statistical analysis. All authors analyzed and interpreted the data. MAI and GVM wrote the manuscript draft. All authors reviewed and edited the manuscript. All authors read and approved the final manuscript.

\section{Funding}

The authors have not declared a specific grant for this research from any funding agency in the public, commercial or not-for-profit sectors.

\section{Data availability statement}

The datasets generated and analysed during the current study are available in the Vivli repository, https://doi.org/10.25934/00006101.

\section{Declarations}

\section{Ethics approval and consent to participate}

The study was conducted under the Declaration of Helsinki and was approved by the Independent Ethical Committee of Istituto Nazionale Tumori IRCCS Fondazione Pascale of Napoli, Italy.

\section{Consent for publication}

Not applicable.

\section{Competing interests}

P.A.A. has/had a consultant/advisory role for Bristol Myers Squibb, RocheGenentech, Merck Sharp \& Dohme, Novartis, Array, Merck Serono, Pierre-Fabre, Incyte, Medimmune, AstraZeneca, Syndax, Sun Pharma, Sanofi, Idera, Ultimovacs, Sandoz, Immunocore, 4SC, Alkermes, Italfarmaco, Nektar, BoehringerIngelheim, Eisai, Regeneron, Daiichi Sankyo. He also received research funding from Bristol Myers Squibb, Roche-Genentech, Array and travel support from MSD. All the other authors declared to have no conflict of interest.

\section{Author details}

1 Division of Laboratory Medicine, Istituto Nazionale Tumori - IRCCS - Fondazione G. Pascale, Naples, Italy. ${ }^{2}$ Unit of Melanoma, Cancer Immunotherapy and Innovative Therapy, Istituto Nazionale Tumori - IRCCS - Fondazione G. Pascale, Naples, Italy. ${ }^{3}$ Epidemiology and Biostatistics Unit, Istituto Nazionale Tumori - IRCCS - Fondazione G. Pascale, Naples, Italy. ${ }^{4}$ Abdominal Oncology Division, Istituto Nazionale Tumori - IRCCS - Fondazione G. Pascale, Naples, Italy. ${ }^{5}$ Department of Breast and Thoracic Oncology, Istituto Nazionale Tumori - IRCCS - Fondazione G. Pascale, Naples, Italy. ${ }^{6}$ Department of Urology and Gynecology, Istituto Nazionale Tumori - IRCCS - Fondazione G. Pascale, Naples, Italy. ${ }^{7}$ Department of Muscle-Skeletal Oncology, Istituto Nazionale Tumori - IRCCS - Fondazione G. Pascale, Naples, Italy. ${ }^{8}$ Department of Thoracic Medical Oncology, Istituto Nazionale Tumori - IRCCS - Fondazione G. Pascale, Naples, Italy. ${ }^{9}$ Head and Neck Medical Oncology Unit, Istituto Nazionale Tumori - IRCCS - Fondazione G. Pascale, Naples, Italy. ${ }^{10}$ Scientific Directorate, Istituto Nazionale Tumori - IRCCS - Fondazione G. Pascale, Naples, Italy. ${ }^{11}$ Department of Oncology-Pathology, Karolinska Institutet, Cancer Centrum Karolinska, 17176 Stockholm, Sweden. ${ }^{12}$ Biostatistic Unit, Istituto Nazionale Tumori Regina Elena, IRCCS, Rome, Italy.

Received: 8 March 2021 Accepted: 19 March 2021

Published online: 31 March 2021

\section{References}

1. van de Haar J, Hoes $L R$, Coles $C E$, et al. Caring for patients with cancer in the COVID-19 era. Nat Med. 2020;26:665-71.

2. Desai A, Sachdeva S, Parekh T, et al. COVID-19 and cancer: lessons from a pooled meta-analysis. JCO Glob Oncol. 2020;6:557-9.

3. Barlesi F, Foulon S, Bayle A, et al. Outcome of cancer patients infected with COVID-19, including toxicity of cancer treatments. In: AACR annual meeting I, April 27-28; 2020.

4. Rugge M, Zorzi M, Guzzinati S. SARS-CoV-2 infection in the Italian Veneto region: adverse outcomes in patients with cancer. Nat Cancer. 2020;8:784-8.

5. Liang W, Guan W, Chen R, et al. Cancer patients in SARS-CoV-2 infection: a nationwide analysis in China. Lancet Oncol. 2020;21:335-7.

6. Zhang L, Zhu F, Xie L, et al. Clinical characteristics of COVID-19 infected cancer patients: a retrospective case study in three hospitals within Wuhan, China. Ann Oncol. 2020;31:894-901.

7. Robilotti EV, Babady NE, Mead PA, et al. Determinants of COVID-19 disease severity in patients with cancer. Nat Med. 2020;26:1218-23.

8. Garassino MC, Whisenant JG, Huang LC, et al. COVID-19 in patients with thoracic malignancies (TERAVOLT): first results of an international, registry-based, cohort study. Lancet Oncol. 2020;21:914-22.

9. Rutigliano JA, Sharma S, Morris MY, et al. Highly pathological influenza A virus infection is associated with augmented expression of PD-1 by functionally compromised virus-specific CD8+T cells. J Virol. 2020;88:1636-51

10. Ethun $C G$, Bilen $M A$, Jani $A B$, et al. Frailty and cancer: implications for oncology surgery, medical oncology, and radiation oncology. CA Cancer J Clin. 2017;67:362-77.

11. Poortmans PM, Guarneri V, Cardoso M-J. Cancer and COVID-19: what do we really know? Lancet. 2020;395:1884-5.

12. Vivarelli S, Falzone L, Grillo CM, et al. Cancer management during COVID19 pandemic: is immune checkpoint inhibitors-based immunotherapy harmful or beneficial? Cancers. 2020;12:2237.

\section{Publisher's Note}

Springer Nature remains neutral with regard to jurisdictional claims in published maps and institutional affiliations. 